Portland State University

PDXScholar

Mechanical and Materials Engineering Faculty

Publications and Presentations

2020

\title{
Data-Driven Modeling of the Wake Behind a Wind Turbine Array
}

\author{
Naseem Ali \\ Portland State University \\ Raul Bayoan Cal \\ Portland State University, rcal@pdx.edu
}

Follow this and additional works at: https://pdxscholar.library.pdx.edu/mengin_fac

Part of the Materials Science and Engineering Commons, and the Mechanical Engineering Commons Let us know how access to this document benefits you.

\section{Citation Details}

Ali, N., \& Cal, R. B. (2020). Data-driven modeling of the wake behind a wind turbine array. Journal of Renewable and Sustainable Energy, 12(3), 033304.

This Article is brought to you for free and open access. It has been accepted for inclusion in Mechanical and Materials Engineering Faculty Publications and Presentations by an authorized administrator of PDXScholar. Please contact us if we can make this document more accessible: pdxscholar@pdx.edu. 


\section{Data-driven modeling of the wake behind a wind turbine array ${ }^{\circledR}$}

Cite as: J. Renewable Sustainable Energy 12, 033304 (2020); https://doi.org/10.1063/5.0004393

Submitted: 12 February 2020 . Accepted: 31 May 2020 . Published Online: 26 June 2020

Naseem Ali (i), and Raúl Bayoán Cal (i)

\section{COLLECTIONS}

EP This paper was selected as an Editor's Pick
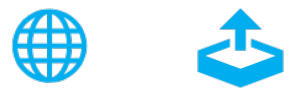

\section{ARTICLES YOU MAY BE INTERESTED IN}

Real-time modeling technique optimizes power output from wind turbines

Scilight 2020, 26111 (2020); https://doi.org/10.1063/10.0001528

Wind and photovoltaic potential in Europe in the context of mid-term energy storage

Journal of Renewable and Sustainable Energy 12, 034101 (2020); https://

doi.org/10.1063/1.5131560

\section{Eulerian CFD model of direct absorption solar collector with nanofluid}

Journal of Renewable and Sustainable Energy 12, 033701 (2020); https://

doi.org/10.1063/1.5144737

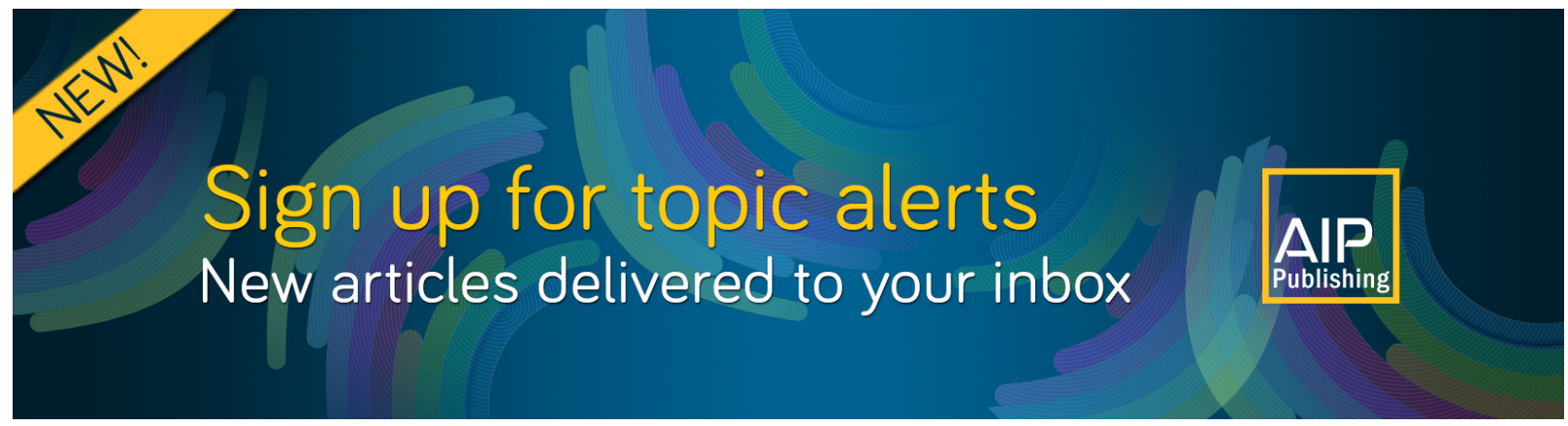




\title{
Data-driven modeling of the wake behind a wind turbine array $\mathbb{B}^{\mathrm{B}}$
}

\author{
Cite as: J. Renewable Sustainable Energy 12, 033304 (2020); doi: 10.1063/5.0004393 \\ Submitted: 12 February 2020 . Accepted: 31 May 2020 . \\ Published Online: 26 June 2020

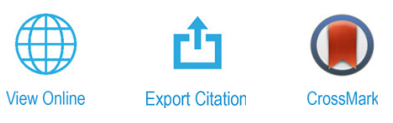

Naseem Ali (iD) and Raúl Bayoán Cal ${ }^{\mathrm{a})}$ (D)

\author{
AFFILIATIONS \\ Department of Mechanical and Materials Engineering, Portland State University, Portland, Oregon 97201, USA
}

a) Author to whom correspondence should be addressed: rcal@pdx.edu

\begin{abstract}
The wake flow in a wind turbine array boundary layer is described using the Koopman operator. Dynamics of the flow are decomposed into the linear and forcing terms, and the low-energy delay coordinates are revealed. The rare events show the non-Gaussian long tails that capture the switching and bursting phenomena. The near-wake region shows the incoherent phase space region, where the dynamics are strongly nonlinear. The far-wake region is marked with the small non-Gaussian forcing term, and the dynamics are largely governed by linear dynamics. The data-driven predictive model is built based on the Hankel-based dynamic mode decomposition and treats the nonlinear state of forcing term as external actuation. The model forecasts the evolution of the flow field for short-term timescales. The mean relative errors between the predictive and test fluctuating velocities are approximately $15 \%$.
\end{abstract}

Published under license by AIP Publishing. https://doi.org/10.1063/5.0004393

\section{INTRODUCTION}

Modeling the turbine response to non-Gaussian conditions can help explain the signature in the aerodynamic forces, fatigue loads, and development of alternative control schemes. ${ }^{1,2,32}$ The variations in the mean statistics of the wind turbine wake flow highlight the nature of the flow response to the external forcing of the wind turbine..$^{2-5,33-35}$ Because turbulence exists on different temporal and spatial scales in a wind farm, the behavior of the non-Gaussian conditions is dependent on the physical location downstream the rotor. ${ }^{2,6}$

Systems like wind plants have a large number of degrees of freedom that cause a considerable amount of uncertainty in parameters when modeling. For wind farm controller analysis, high fidelity models are required for exploring the optimal possibilities of wind farm control. Due to the computational cost of these models, they are not convenient for pursuing a dynamical system and control model. Therefore, new generations of models are required to solve this deficiency. Reduced-order models can provide coherent features of wake dynamics and solve part of the computational complexity although these models are only suitable for specific conditions and are not proven for real cases. Linking between reduced-order model and parameter-varying control can provide a new generation of the optimized wind farm control.

Developing a flow model from statistics based on the ensemble of observations, which can represent a solution to the dynamical system itself, is of central importance. The data-driven model includes reducing the degrees of freedom and presenting a dynamical model that links the evolution of the most dominant features and the trajectories in the high-fidelity phase space. The wake of the turbine is a nonlinear system with intermittent energy that transfers between flow scales in the energy cascade. ${ }^{7,8}$ Therefore, predicting and identifying the coordinate transformations that highlight the time response to the strongly nonlinear forces are potentially important for the wind farm design. Time evolution of interacting quantities in engineering applications such as wind energy can be described using a dynamical system approach.

Data-driven techniques have recently received more attention as alternative approaches for directing wind farm analysis or control. These approaches rely on specific operating conditions; hence, they provide linear models for a specific operating point. ${ }^{9}$ Because the operation of the wind turbines is time dependent, data-driven approaches can describe the wake in more detail regarding the flow conditions, such as thermal stability and turbulent intensity, as well as provide systems the ability to automatically learn and improve performance and stability. Following the Takens embedding theorem, which translates high-dimensional datasets into a relatively low-dimensional space, applied to a sequence of observations of the state, data-driven dynamical systems can detect the full dynamics by reconstructing a set of states and preserve the structural aspect of the states. ${ }^{10}$ The intermittent forces are strange phase spaces of a dynamical system characterized by chaotic trajectories, and are responsible for divergent behavior 
with time and cause uncertainty to the prediction. Consequently, isolating the deterministic structure from the randomness of the chaotic dynamics is potentially useful for control strategies. Koopman operator theory is an efficient approach to decompose the chaotic system into the linear evolution and nonlinear forcing, where the time history of the measurements can estimate the full-state of the system and provide a foundation for its linear dynamics.

In nonlinear dynamics, the dynamical system can be represented in the linear representations through the Koopman analysis. A Koopman operator has the ability to analyze and tackle the nonlinear system in an infinite-dimensional linear approach. ${ }^{11}$ In data-driven models, for example, Giannakis and Majda ${ }^{12}$ and Giannakis ${ }^{13}$ projected the delay-embedded data onto the basis function of Laplace-Beltrami and Koopman operator respectively, to present a model reduction with the aim of forecasting the time series for ergodic dynamical systems. Arbabi and Mezic ${ }^{14}$ linked the Hankel based dynamic mode decomposition (DMD) to the Koopman operator, demonstrating that applying DMD to Hankel observation matrices yields the true Koopman eigenvalues and eigenfunctions. Korda and Mezić ${ }^{15}$ identified linear predictors for nonlinear controlled dynamical systems using the Koopman operator framework. The nonlinear dynamics are projected to a higher dimensional space that displays the evolution is approximately linear. Giannakis et al. ${ }^{16}$ employed a Koopman eigenfunction analysis to study the long-time evolution of the three-dimensional flow. Their findings showed that the behavior of the large-scale circulation is recovered from the eigenfunctions of a data-driven Koopman operator. Brunton et al. ${ }^{10}$ provided a Hankel alternative view of Koopman to understand the disorder in chaos quantitatively by treating examples such as measles outbreaks and magnetic fields. Recently, Khodkar et al. ${ }^{17}$ introduced the Koopmanbased framework for forecasting the spatiotemporal evolution of highdimensional chaotic dynamics and turbulence.

Data-driven approaches have been used to study wind turbine wakes. For example, Gebraad et al. ${ }^{18}$ presented a novel data-driven model to optimize the yaw settings of wind turbines in a wind plant for improved energy production of the wind plant. Iungo et al. ${ }^{19}$ proposed a data-driven RANS approach to optimize power plant control and prevent detrimental wake interactions. Iungo et al. ${ }^{20}$ proposed a new data-driven reduced order model for prediction of wind turbine wakes. The model captured the main physical processes and dynamics of wind turbine wakes. Park et al. ${ }^{21}$ used a data-driven optimization method to find the optimum control actions. Göçmen and Giebel ${ }^{22}$ presented a data-driven wake model for short-term power estimation. $\mathrm{Ti}$ and $\mathrm{Yang}^{23}$ used the machine learning and computational fluid dynamics simulation to develop new wake velocity and turbulence models to improve the turbine wake predictions.

Data-driven analysis based on the Koopman operator is used here to build a linear model in the leading delay coordinates to forecast the future state of stochastic fluctuating velocity. The model will be built after isolating the linear modes from a low energy delay coordinate that triggers intermittent bursting and switching events. The nonlinear term is used as an input parameter to train the model. The aim is also to connect the nonlinear mode distribution, which causes the linear model to diverge, with intermittency, and quantify the activity of the intermittent force with respect to time. The manuscript is organized as follows: Sec. II outlines the Koopman theory; Sec. III presents in detail experimental setup; Sec. IV provides a discussion of the finding and the relevance in the field; Sec. V presents the forecasting for the spatial and temporal evolution. Conclusions are presented in Sec. VI.

\section{THEORY}

The perspective of the Koopman operator relies on mapping the measurement dataset to different coordinates that make the dynamics appear linear. Here, the Koopman operator is built based on the Hankel matrix, $H$, as ${ }^{10}$

$$
\begin{aligned}
H & =\left[\begin{array}{cccc}
u\left(t_{1}\right) & u\left(t_{2}\right) & \cdots & u\left(t_{p}\right) \\
u\left(t_{2}\right) & u\left(t_{3}\right) & \cdots & u\left(t_{p+1}\right) \\
\vdots & \vdots & \ddots & \vdots \\
u\left(t_{q}\right) & u\left(t_{q+1}\right) & \cdots & u\left(t_{m}\right)
\end{array}\right] \\
& =\left[\begin{array}{cccc}
u\left(t_{1}\right) & \mathcal{K} u\left(t_{1}\right) & \cdots & \mathcal{K}^{p-1} u\left(t_{1}\right) \\
\mathcal{K} u\left(t_{1}\right) & \mathcal{K}^{2} u\left(t_{1}\right) & \cdots & \mathcal{K}^{p} u\left(t_{1}\right) \\
\vdots & \vdots & \ddots & \vdots \\
\mathcal{K}^{q-1} u\left(t_{1}\right) & \mathcal{K}^{q} u\left(t_{1}\right) & \cdots & \mathcal{K}^{m-1} u\left(t_{1}\right)
\end{array}\right],
\end{aligned}
$$

where $\mathcal{K}$ is the Koopman operator, $u$ is a vector-valued observation of fluctuating velocity, and $t$ is the time. Singular value decomposition (SVD) of the Hankel matrix can estimate the embedding dimension for time-delay coordinates that provide the conditions under which a chaotic dynamical system is reconstructed from a set of observations of the state of a dynamical system as

$$
H=\Psi \Sigma V^{T},
$$

where the columns of $\Psi$ and $V$ present the right- and left-singular vectors, respectively; they are a set of orthonormal eigenvectors. The chaotic dynamics is decomposed using the Hankel alternative view of Koopman into a linear model and the forcing term. The eigentimedelay vector, $V=\left[v_{1}, v_{2}, \quad \ldots \quad, v_{R-1}\right]^{T}$, is used to define the forced term $v_{R}$ from the nonlinear dynamics. Following the Hankel alternative view of Koopman approach introduced in the work of Brunton et al. $^{10}$ and Khodkar et al., ${ }^{17}$ adding a forcing term to the linear model is considered to approximate the nonlinear dynamics more accurately. A future vector-valued observable that is not a part of the training data can be predicted as

$$
u^{t+1}=A u^{t}+B F,
$$

where $F$ is the form of external forcing, where it takes the quadratic form based on the Reynolds stress terms. The $B$ is the control matrix related to the forcing term. The $A$ and $B$ are the input parameters to create state-space model and simulate the time response of dynamic system, respectively. The Hankel based dynamic mode decomposition with control (H-DMDc) is used to estimate the $A$ and $B$ matrices using the minimization approach introduced in the work of Proctor et al., ${ }^{24}$

$$
\begin{gathered}
A=\Psi^{T} \Pi V \Sigma^{-1} \Psi_{1}^{T} \Psi, \\
B=\Psi^{T} \Pi V \Sigma^{-1} \Psi_{2}^{T},
\end{gathered}
$$

where these matrices are calculated in a reduced-dimension subspace. Here, $\Psi_{1}$ and $\Psi_{2}$ are constructed from the first $q m$ and the remaining rows of $\Psi$. Figure 1 summarizes the main steps of the current approach. The training data that are obtained from any velocity or power sensor can be fed into DMDc algorithm to estimate the model 


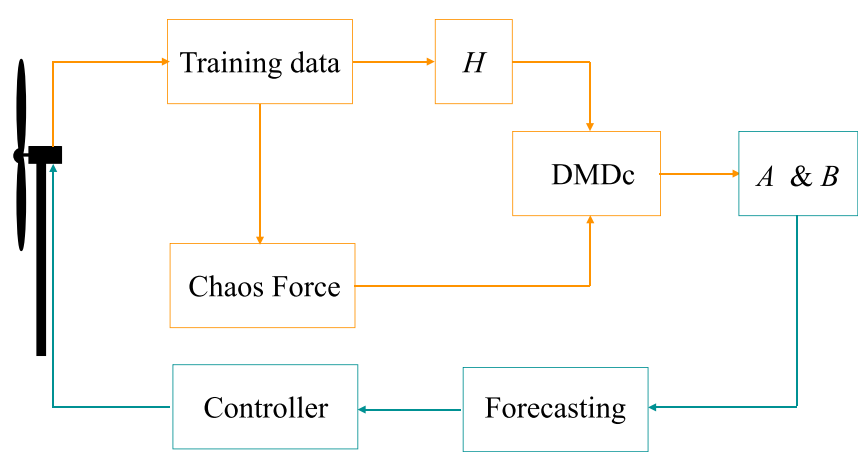

FIG. 1. Schematic of data-driven method.

operators. The forecasting step starts after as shown in Eq. (3). The advantage of forecasting step can be fed into a controller to change the turbine condition.

\section{EXPERIMENTAL SETUP}

An experiment with an array of horizontal axis wind turbines was conducted at the closed-loop Corrsin wind tunnel at Johns Hopkins University. The entrance of the tunnel was furnished with an active grid to generate turbulence. The inlet mean velocity shear profile was generated using nine strakes. A 24-grit aluminum oxide sand paper was provided along the test section in the ground to roughen the boundary shape, see Fig. 2. A three-bladed rotor of $12 \mathrm{~cm}$ diameter, $D$, was used as a wind turbine model. Nine wind turbines models were distributed as a $3 \times 3$ array with space of $7 \mathrm{D}$ in the streamwise and $3 \mathrm{D}$ in the spanwise directions. The measurements considered here were taken behind the center of the third row of the wind farm. The data were gathered at a frequency of $40 \mathrm{kHz}$ to sample $4 \times 10^{6}$ data points. The measurements were conducted at the streamwise-wall-normal plane $(x-y)$ and the spanwise-wall-normal plane $(z-y)$. For the $x-y$ plane, the measurements were collected at nine locations in the streamwise direction starting from $x=0.5 D$ to $x=8 D$. For the $z-y$ plane, the measurements were taken at nine locations in the spanwise direction starting from $z=-4 D / 6$ to $z=4 D / 6$ with an increment of $D / 6$. For each plane, the measurements are taken for 21 locations in the wall-normal direction starting at $0.5 \mathrm{~cm}$ above the ground and move vertically with an increment of $1 \mathrm{~cm}$. More details about the experimental setup and measurements can be found in the work of Cal et al. $^{2}$

\section{RESULTS}

Statistical quantities are shown here to provide an important distinction between the flow regions downstream the rotor. Figure 3 presents the normalized streamwise mean velocity $\left(U / U_{\infty}\right)$, and the ratio between the integral length scale $\left(L_{i n}\right)$, and Taylor microscale $(\lambda)$. The integral length scale is calculated based on auto-correlation between two different times. The impact of the rotor is substantial on the near-wake region, specifically behind the nacelle and tower, where the minimum values of the streamwise mean velocity, integral length scales, and Taylor microscale are observed. These parameters monotonically increase toward the outside of the rotor. The same trend is shown in the comparison between the near- and far-wake regions. The tower adds more perturbations to the flow and translates to the wake region. In the spanwise direction, the wake expansion is shown below the top tip (TT), where velocities are low. The reduced size of the $L_{i n}$ is due to the amplification of enstrophy and flow separation. The flow outside the swept area is marked with relatively constant $L_{i n}$ and $\lambda$, specifically above the top tip.

Figure 4 presents the principal components of the SVD with the linear model that is built based on the regression model for the hub height $(\mathrm{HH})$ and top tip locations at the center of the rotor. The figure shows the main principle of the Koopman analysis using a SVD in a finite-dimensional nonlinear system by testing the quality of the reconstruction of the eigentime series. These locations are shown for generality as they present the most sensitive locations that carry chaotic changes in the wake. The regression model is presented for the linear variables in right-singular vectors $V$. Then, the regression coefficients for linear and forcing terms are used to train and simulate the time response of the dynamical system. The Hankel decomposition can analyze complex time series and reveal the characteristic features of strongly nonlinear dynamics (rare events). For both cases, the linear model fits and captures the main features in the transition coordinate. The simulated trajectory using the fit model matches that of the original system, indicating the well conditioning. The variance of the distributions at the peaks indicates the small errors in the estimation of the eigenvectors. The dynamics of the linear model can predict the spatialtemporal evolution after training the model. The model use the forcing term as an input to run the on-line model and provide an accurate reconstruction of the new attractor corresponding to the test data.

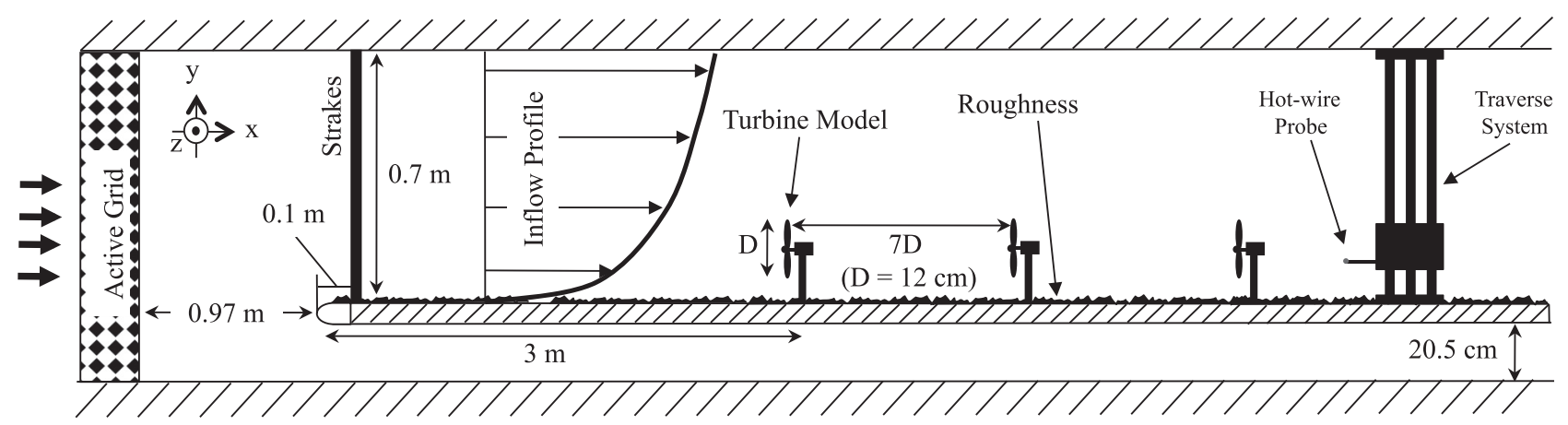

FIG. 2. Experimental setup. Reproduced with permission from Cal et al., J. Renewable Sustainable Energy 2(1), 013106 (2010).$^{25}$ Copyright 2010 AIP Publishing. 
(a) $U / U_{\infty}$
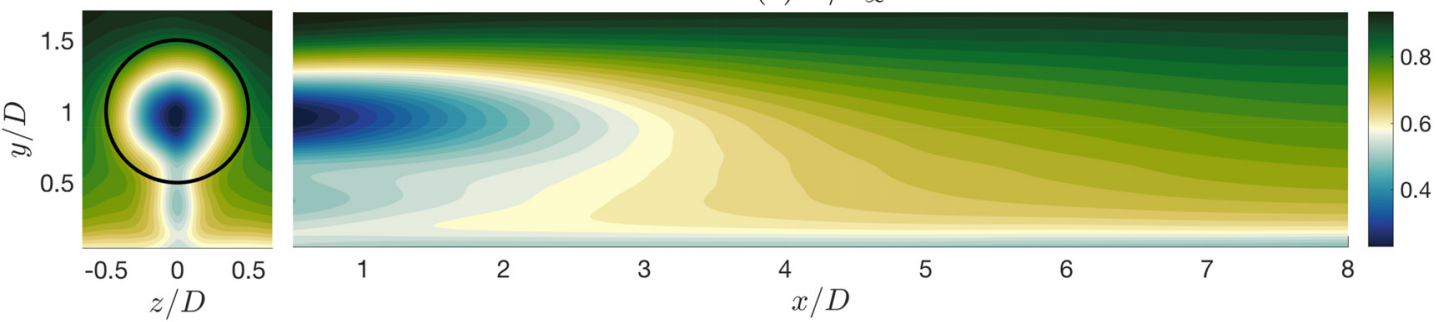

(b) $L_{\text {in }} / \lambda$
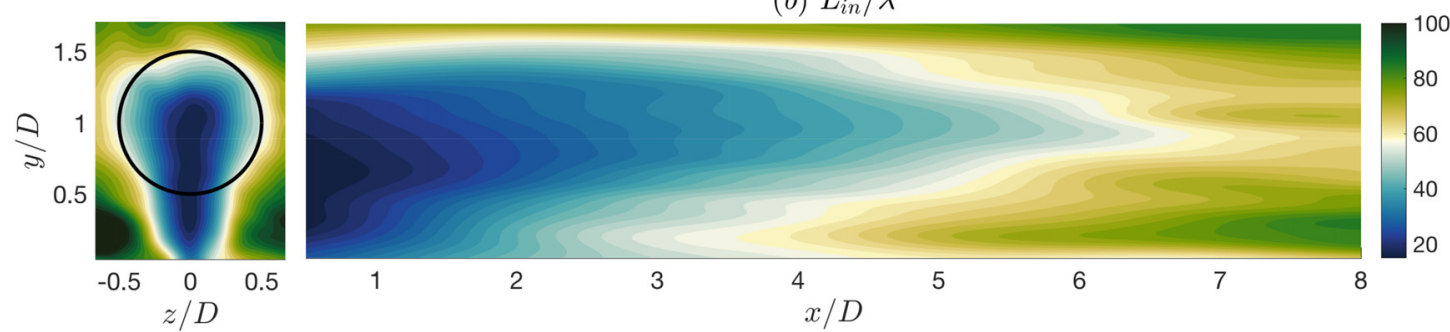

FIG. 3. Flow characteristics: (a) normalized mean velocity $\left(U / U_{\infty}\right)$, (b) the ratio between the integral length scale $\left(L_{i n}\right)$ and Taylor microscale $(\lambda)$. The black circle indicates the rotor area.

The statistics of the forcing term $v_{R}$ are a non-Gaussian distribution with long tails as shown in Fig. 5. The most active place regarding the forcing term is noticed at the hub height. In contrast, the far-wake region shows a small departure from Gaussianity. Thus, the $v_{R}$ carries the signature of high-frequency bursts and it is important to find the timing of this forcing to characterize the switching dynamics. Characterizing the times of the nonlinear force is required to uniquely determine the future system states and isolate the impact of the external forces with respect to a background flow. Linear system identification is applied in the literature in many applications such as in climate time series analysis. ${ }^{26,27}$ The instability and intermittent phenomena generate a variable dynamic loading and fatigue as well as have a major impact on the stability of the grid. In physical space, the intermittent events correspond to the rotor swept region that introduces most perturbations to the energy cascade. The transition of these events through the velocity signal and the advection on the nonlinear force downstream the rotor are still unknown.

(a) $\mathrm{HH}$

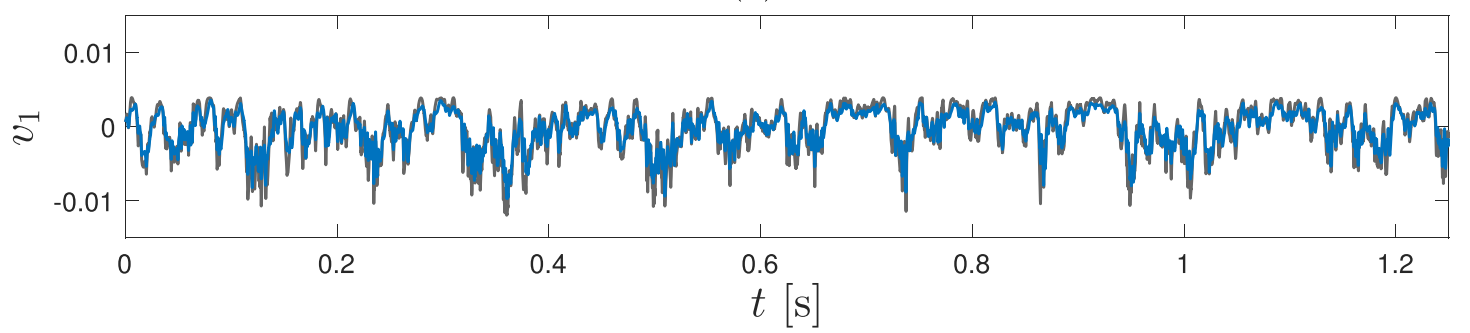

(b) $\mathrm{TT}$

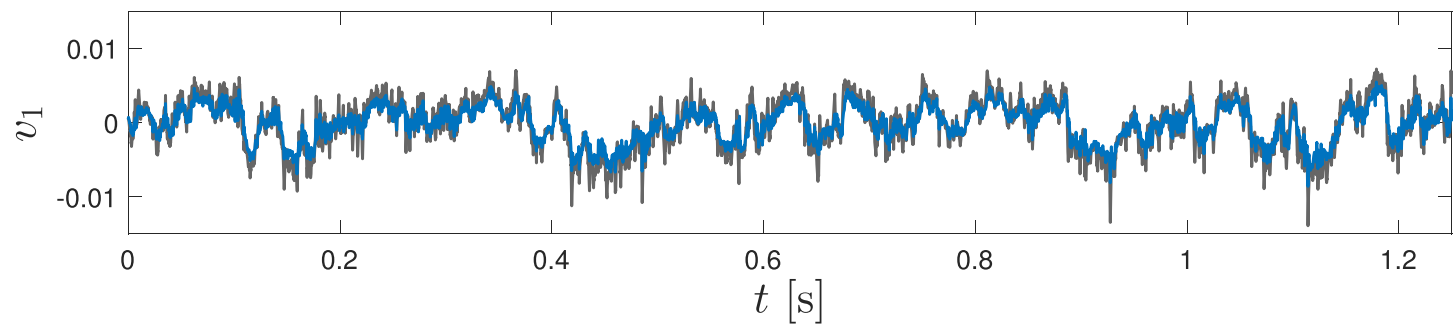

FIG. 4. The eigentime-delay series (gray) with the linear model of the flow at the hub height $(\mathrm{HH})$ and top tip (TT) locations of $z=0$. 

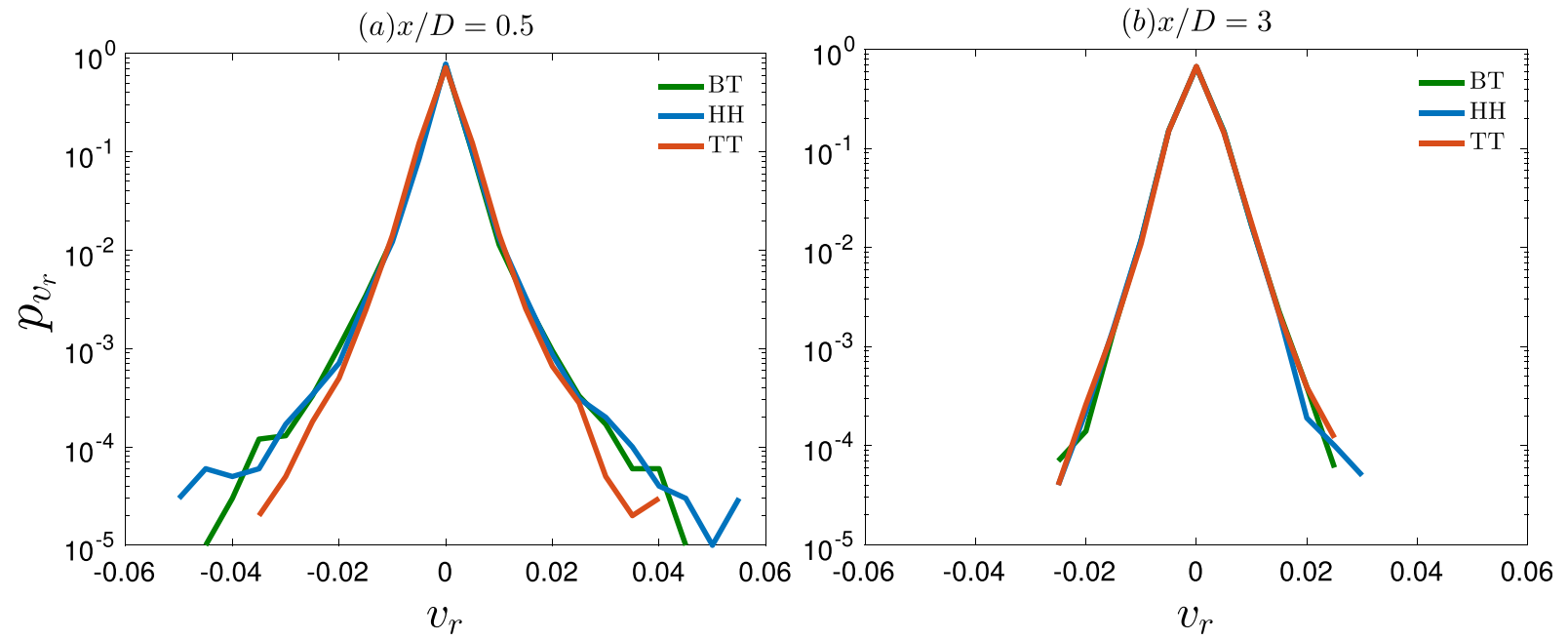

FIG. 5. Probability density function (PDF) of the rare forcing events at the bottom tip (BT), hub height (HH), and top tip (TT) of $z / D=0$ location.

Figure 6 presents the principal components of the SVD for the hub height location including the linear term, $v_{1}$, and the nonlinear forcing term, $v_{R}$. To uncover the locations at which the nonlinear forcing term is active, the $v_{R}$ is isolated above a certain threshold. The locations that show inactive force are marked with black color. The eigentime-delay vectors gradually develop structures and spectral content. Forcing term $v_{R}$ is the nonlinear geometrical structure of time series data and presents the existence of intermittent patterns in the considered locations. During the intermittent bursts, the nonlinear modes display the activated fine spatial structure and show the departure from Gaussianity. Note that the dynamics of the trajectories are governed by the linear term when the forcing term is small. The hub height location at the rotor center shows relatively large fluctuations in the nonlinear term in comparison with that at $z / D=4 / 6$. The center of the rotor presents the nonlinearity as an on-off intermittent force. Switching between chaotic bursts static behavior can describe the nonlinearity of the system. Platt et al. ${ }^{28}$ highlighted that on-off chaos can be detected at the suitably chosen coordinates. Using the Hankel matrix analysis provides the opportunity to classify the flow signal and highlights these coordinates that show the alternating in the chaosity. The locations downstream the rotor have unstable invariant manifolds and attractors that produce on-off intermittency. Outside the swept area, the nonlinearity displays less switching with the time and the activity of the force is balanced by inactivity periods, indicating that linearity suppresses the forcing terms and the flow becomes less chaotic. The statistics of the forcing term activity at the hub height show that at the center of the rotor the activity presents about $68 \%$ of the total time duration, whereas outside the swept region the activity presents about $78 \%$. Although the ratio activity of the forcing term outside the swept area is larger than inside, the strength of the nonlinearity is much small outside the rotor area, indicating that the large forcing term induces the flow to react against the nonlinear forces and makes a balance to null the nonlinearity.

Figure 7 presents the principal components of the SVD for the top tip location including the linear and the nonlinear forcing terms.
The physical location shows less impact on the flow at the top tip and above, where the inside and outside the swept area show the same trend in the nonlinearity distribution through the forcing term. As pointed in Ali and $\mathrm{Cal},{ }^{29}$ the top tip location is less intermittent and independent of the physical location. Here, the same trend is shown regarding location independence. However, the flow at these locations carries a nonlinear force as shown in Fig. 7. The justification for this observation is that the nonlinearity of the forcing term can take different shapes; one of them is the intermittency. Thus, the nonlinearity can be imposed by a different mechanism that is active at these locations, for example, the tip vortices, momentum entrainment, and the interaction between the wake and the flow above. This is the extension for all approaches that are used to detect the intermittency in the energy cascade of turbulence. The statistics of the forcing term activity at the top tip display that at the center of the rotor the activity presents about $75 \%$ of the total time duration, whereas the activity at the outside of the swept region is about $79 \%$, indicating that the chaos induced by the wind turbines is dependent on the parameter variations and the external disturbances change percentage of nonlinearity to a certain degree. A small variation in the activity is presented in the top tip and above regions. Also, in comparison with the hub height, the activity is substantial at the top tip but the amplitude of the force is low.

Figure 8 presents the principal components of the SVD for the hub height position at two locations downstream the rotor, including $x / D=1$ and 8 . This figure is introduced to check the transition and the activity of the nonlinear forcing term downstream the rotor. The shape of the nonlinear term reveals the signature of the burst events. The on-off forcing is dominant in the near-wake region and distributed at a certain frequency with a high level of nonlinearity. Moreover, the activity of the forcing term is pronounced when the linear term is negative, indicating that the intermittent events are starker at lowspeed flow. Also, the trajectories of the nonlinear forcing term reveal that the flow dynamic at the near-wake region follows different trajectories, where they never exactly are repeated. Moving from $1 D$ to $8 D$ 
$(a) z / D=0$
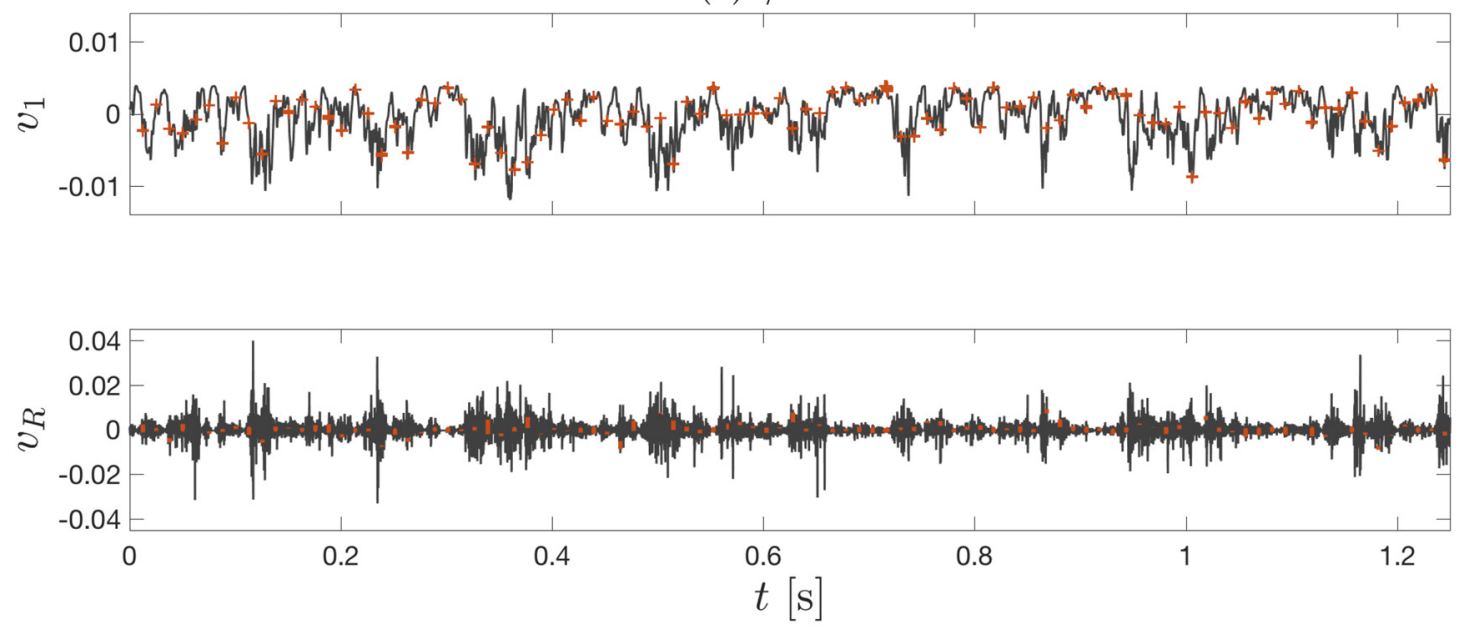

(b) $z / D=4 / 6$
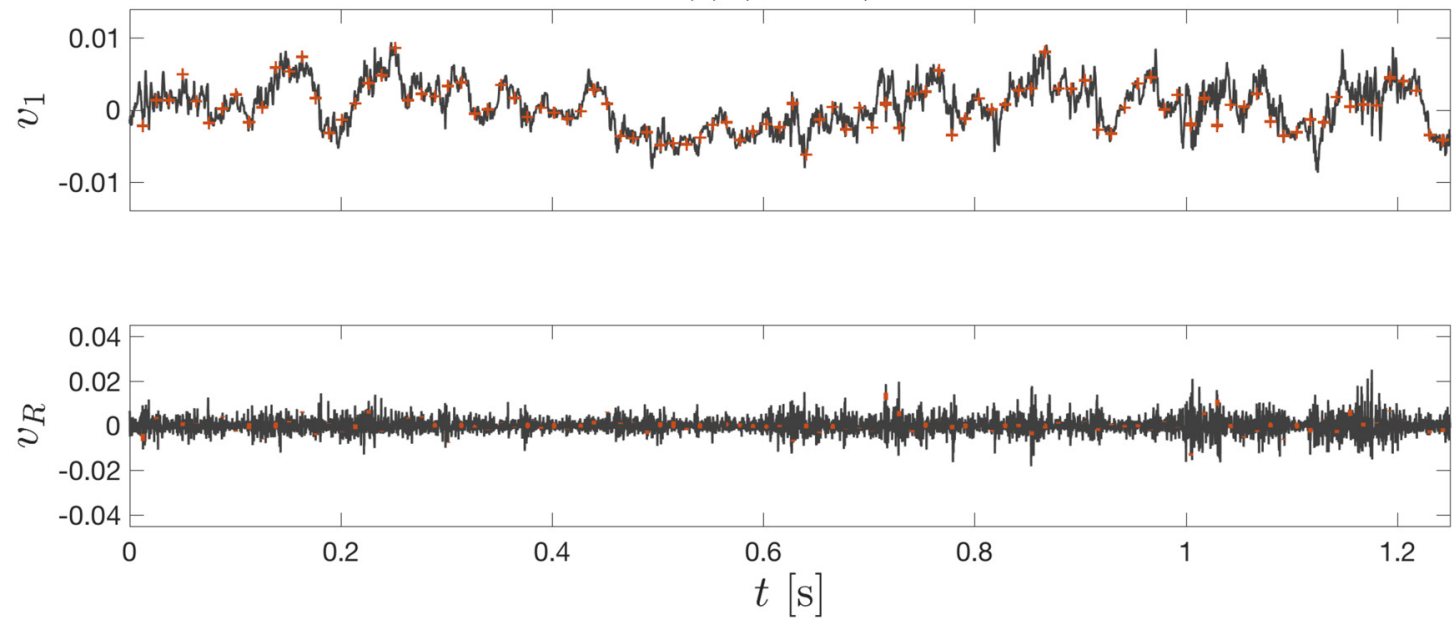

FIG. 6. The eigentime-delay series and forcing term of the velocity signal at the hub height location of $z-y$ plane.

reduces the frequency of the burst events and also shrinks the amplitude of the nonlinearity. Thereafter, the flow at the far-wake region $x=8 D$ shows a major reduction in the burst event and the nonlinearity is uniformly distributed along with the linear term. The statistics of the forcing term activity reveal that at the near-wake region the activity presents about $75 \%$ of the total time duration, whereas outside the swept region the activity presents about $78 \%$. It is interesting to observe that the ratio at the far-wake region is the same ratio of the forcing terms outside the swept area at the $z-y$ plane.

The statistics of the principal components describe the velocity fluctuation distribution based on the physical location within the turbine canopy and show the greatest amounts of variability. In general, the near-wake region displays more nonlinear behavior due to the tip vortex shedding associated with that location. The swept area of the rotor is imprinted with different sizes of coherent structures caused by the nacelle, tower, and rotor tips that consequently alter the inertial subrange of the energy cascade via the shedding frequency. Vortex shedding changes the flow structure and imposes highly anisotropy near the rotor. The anisotropy is also influenced by the turbulence kinetic energy production and the entrainment from above the canopy, thus increasing the interaction between the scales and introducing the on-off intermittent events. ${ }^{30,31}$ On the other hand, the far-wake region is marked with small amplitude intermittent forces.

\section{FORECASTING SPATIAL-TEMPORAL EVOLUTION}

The main goal of approximating the Koopman operator is to delineate all of the behavior of the nonlinear system in the stability region of the dynamical systems and linearize the nonlinear dynamics. The required training and testing sets are reconstructed from the time signal of the measurement points described in Sec. III. The prediction is achieved for thousands of time steps. Each time step presents an average of $0.03 \mathrm{~s}$ (1200 time increments), which is approximately the order of the integral length scale. Treating the flow as a linear model with the explicit interpretation of the nonlinearity as an external force 
$(a) z / D=0$
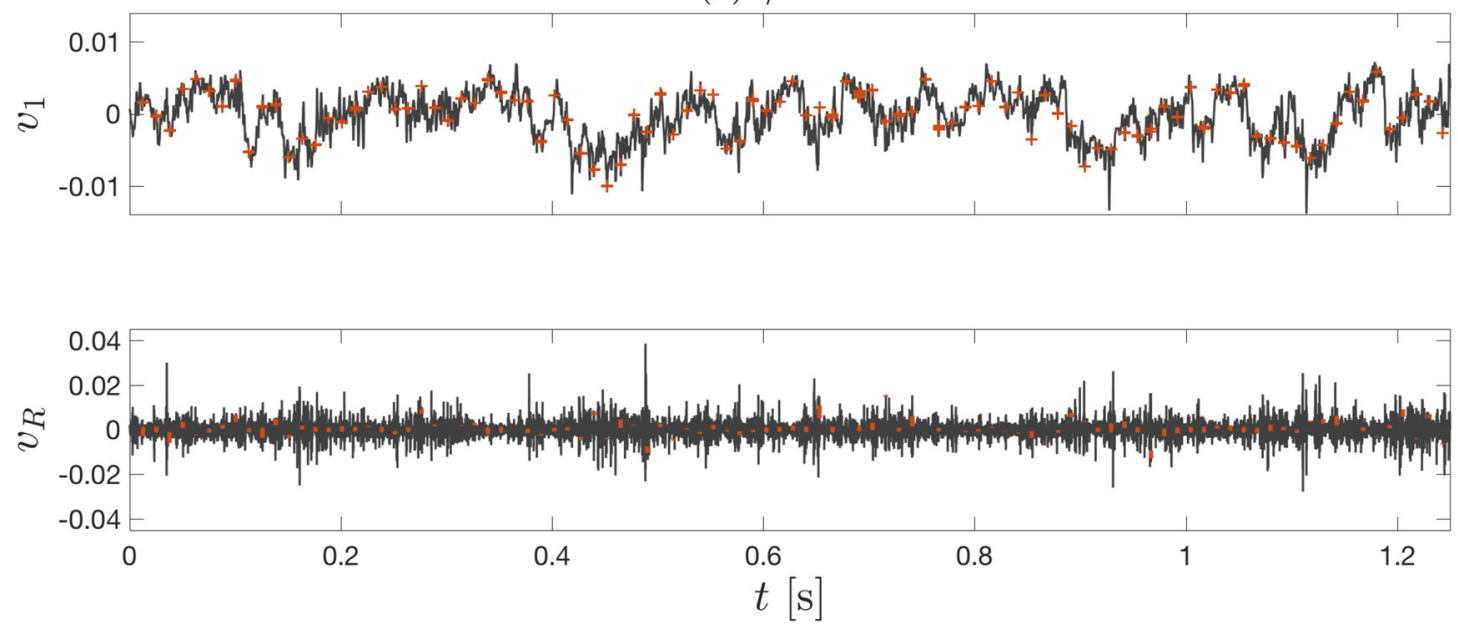

(b) $z / D=4 / 6$
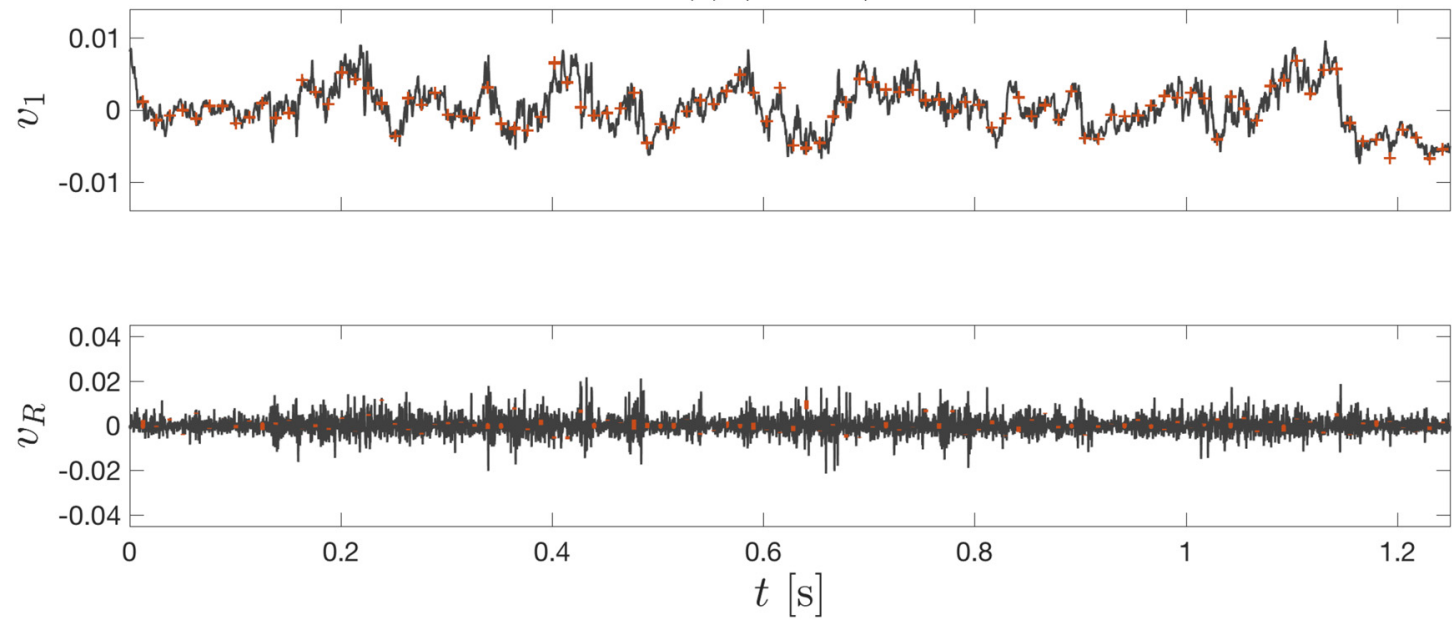

FIG. 7. The eigentime-delay series and forcing term of the velocity signal at the top tip location of $z-y$ plane.

helps in presenting a promising model for prediction of the underlying nonlinear processes. Figures 9 and 10 show the prediction of the fluctuating velocity signals of 21 wall-normal locations at different places downstream the rotor. The prediction matches the test data in a short time and starts diverging from the testing data relatively short times, meaning the underlying dynamics for detecting the nonlinearities are discovered through the data-driven approach. However, the divergence shown after a short time is due to that the delay-embedding of the measurements are not provided any information about the order of nonlinearity. The mean relative error is defined as $E(t)=\left\|u_{f}^{P_{r}}-u_{f}^{\text {test }}\right\|_{2} /\left\|u_{f}^{\text {test }}\right\|_{2}$, where $u_{f}^{P_{r}}$ is the fluctuating velocity that is predicted from the model, and $u_{f}^{\text {test }}$ is the actual fluctuating velocity measured via the hot-wire anemometer. The mean relative errors are approximately $15 \%$, where less accurate prediction is shown with increasing advective timescales, where the growth becomes exponentially unbound.
The proposed tool for the prediction can be part of the wind farm control system to predict the wind and control the turbines such as changing the yaw angle of the wind turbine or the blade pitch to keep optimal operating conditions for all turbines in the array, and that will ensure the increase in the power output. The predicted model is a critical key for model predictive control, where the switching control between a number of actuation (for example, yawed and nonyawed) can be achieved to track a reference operation in an unsteady flow within wind farm. ${ }^{18}$ The turbine sensors used in the field such as sensors that measure power or LIDAR signals can be used as an input to achieve the wind farm control. ${ }^{9}$ The Koopman operator can be used as a systems model to process noise and measurement noise via two steps: first, the prediction step that utilizes a previously estimated state of the linear model to predict the value of the next state and estimate the state covariance. The second step is the updated step, where the state estimation will be corrected based on the current 
(a) $x / D=1$
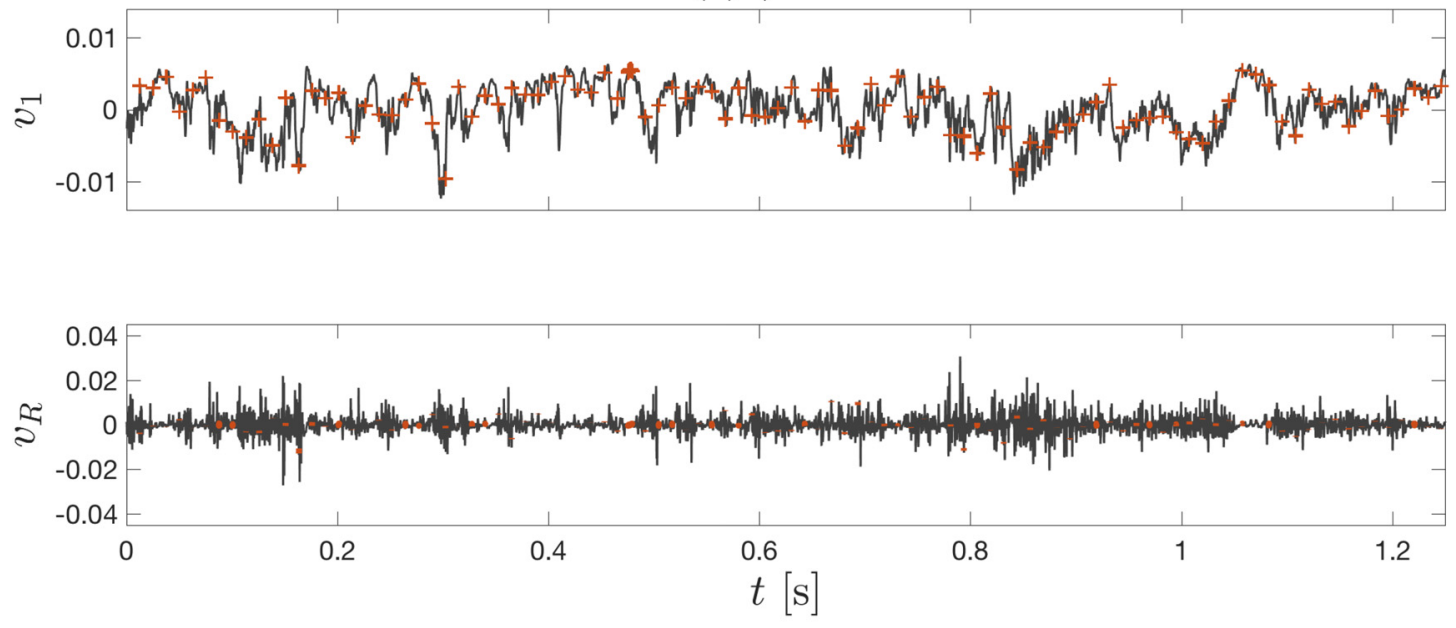

(b) $x / D=8$
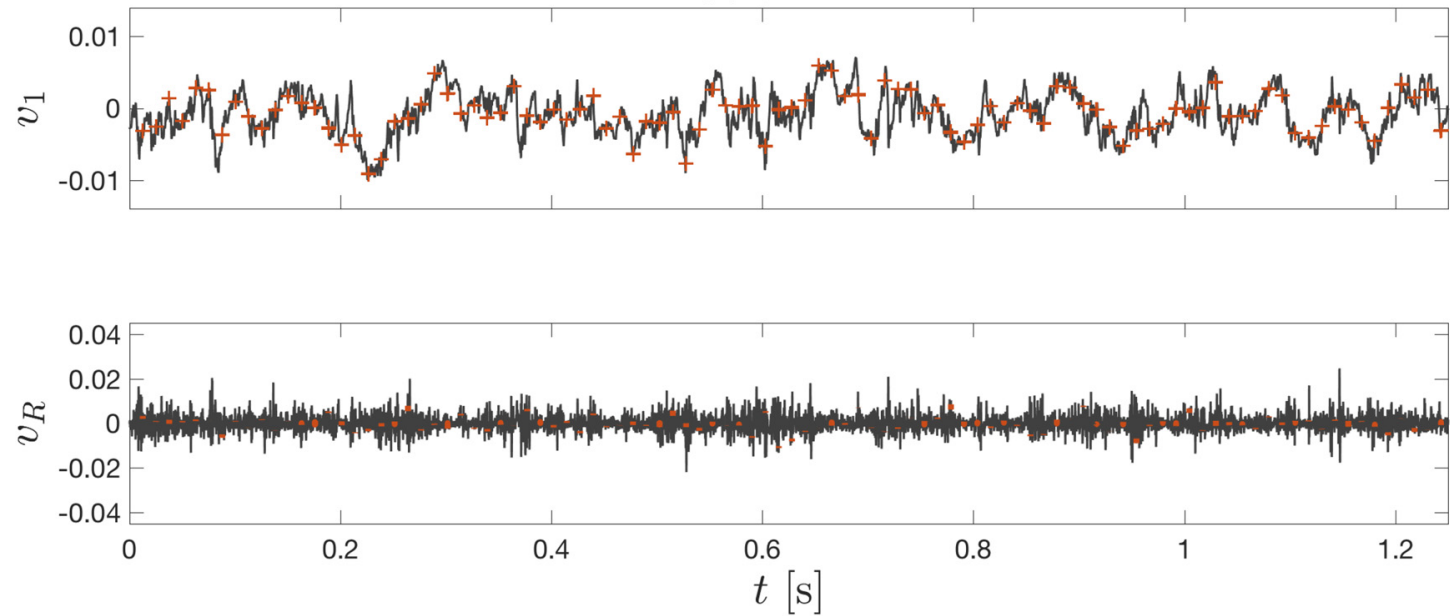

FIG. 8. The eigentime-delay series and forcing term of the velocity signal at the hub height location of $x-y$ plane.

measurements and the statistical properties of the model. The evolution of the error covariance can be predicted with the full state forward model, where the actual observations will be used as an input into mathematical and computational models to create a unified, complete description. Also, this method can be used to model the dynamic stability of the grid in response to disturbances.

\section{CONCLUSION}

The chaos of the wake flow behind a wind turbine is presented using the Koopman operator. The goals of the current work are to connect the chaos force, which causes the linear model to diverge, with intermittency, demonstrate, and quantify the activity of the intermittent force with respect to time; and more importantly, evaluate the proficiency of the data-driven model to predict the future state of the system, where the stochastic fluctuating velocity is based on trained data.
Linear representations show the potential ability to distinguish flow events downstream the rotor. The major variations in the nonlinear forcing term are found at the center of the rotor, where the hub height shows the large transients and intermittent events. Less nonlinearity is shown at the top tip location. Outside the swept area, the locations share the same nonlinearity events. Also, the signature of the nonlinear forces sustains outside the rotor area. The robustness of the nonlinear forcing term is shown outside the swept area and at the farwake regions. This model physically describes the chaotic transitions between the center of the rotor and the atmospheric stream flow, caused by wake advection and vortex shedding in the spanwise direction. The current approach also successfully predicts the flow field for short term timescales. The mean errors between the predictive and test fluctuating velocities are approximately 15\%. The proposed method needs further investigations to obtain a full description of the nonlinear term. Achieving this goal will help in forecasting the variability of 
(a)

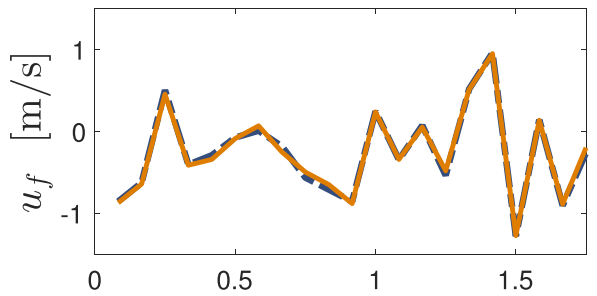

(c)

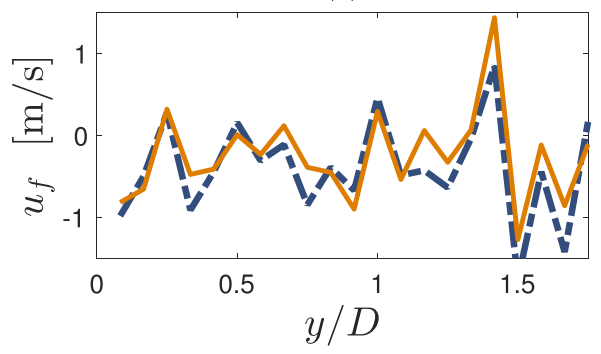

(a)

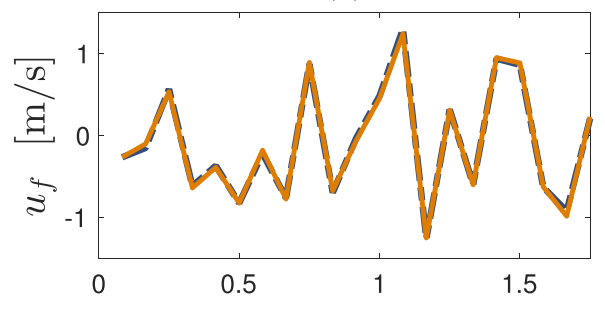

(c)

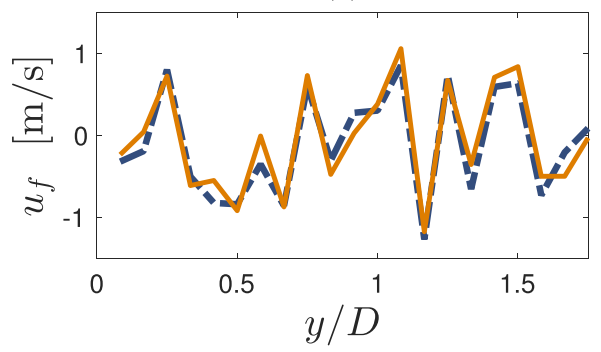

(b)

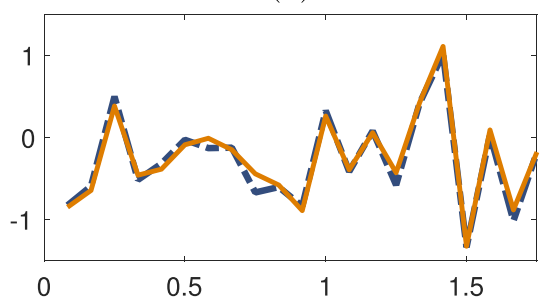

(d)

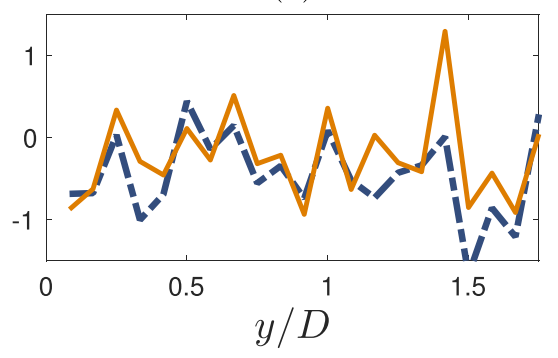

(b)

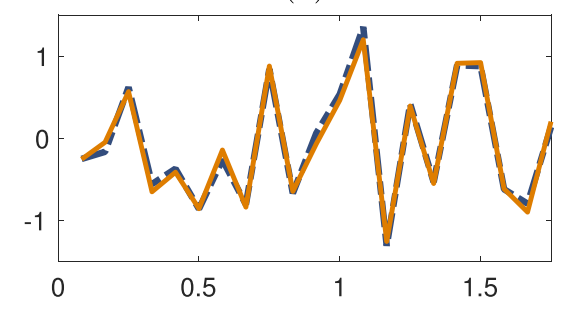

(d)

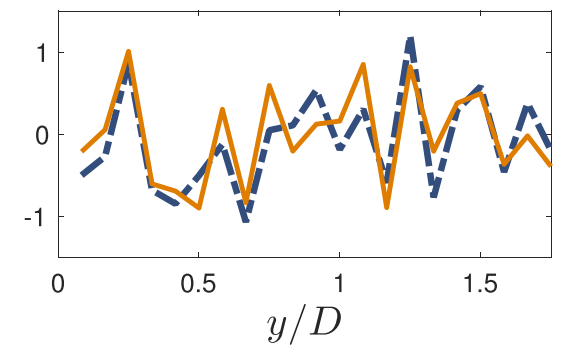

FIG. 9. Predication fluctuating velocities at $x / D=1$ at (a) 75 , (b) 150 , (c) 450 , and (d) 800 time units. The blue and orange lines present the measured and predicted velocities, respectively.
FIG. 10. Predication fluctuating velocities at $x / D=6$. the wind to protect the system in high winds, capture more energy in low winds, and reduce the uncertainty of the output by supporting real-time operations. Forecasting the variability of the wind will allow anticipating wind generation levels to adjust the power system accordingly.

The current model is a part of system identification and uses the measurements from the wind plant to estimate the relevant properties of the system. The simple structure and fast computation of the datadriven model are well-suited for real-time control. The measurements of the real-world wind plant can be fed into the model structure to identify the model parameters and then adjust the internal model based on the predicted future state and test a few sets such as yawed angles to reach the optimized control points. Based on the evaluation of predicted turbine power outputs, the updated yaw settings are stored as a baseline if improvement is shown. Another advantage of the current model is that it can display the effect of inflow conditions and tested parameters on the relevant structural loads on the turbine by analyzing the nonlinear forces.

\section{DATA AVAILABILITY}

The data that support the findings of this study are available from the corresponding author upon reasonable request. 


\section{REFERENCES}

${ }^{1} J$. Schottler, N. Reinke, A. Hölling, J. Whale, J. Peinke, and M. Hölling, "On the impact of non-Gaussian wind statistics on wind turbines-An experimental approach," Wind Energy Sci. 2(1), 1-13 (2017).

${ }^{2}$ N. Ali, A. Fuchs, I. Neunaber, J. Peinke, and R. B. Cal, "Multi-scale/fractal processes in the wake of a wind turbine array boundary layer," J. Turbul. 20(2), 93-120 (2019).

${ }^{3}$ N. Ali, N. Hamilton, M. Calaf, and R. B. Cal, "Turbulence kinetic energy budget and conditional sampling of momentum, scalar, and intermittency fluxes in thermally stratified wind farms," J. Turbul. 20(1), 32-63 (2019).

${ }^{4}$ R. Scott, B. Viggiano, T. Dib, N. Ali, M. Hölling, J. Peinke, and R. B. Cal, "Wind turbine partial wake merging description and quantification," Wind Energy 23(7), 1610-1618 (2020).

${ }^{5}$ H. Kadum, R. B. Cal, M. Quigley, G. Cortina, and M. Calaf, "Compounded energy gains in collocated wind plants: Energy balance quantification and wake morphology description," Renewable Energy 150, 868-877 (2020).

${ }^{6}$ R. Calif and F. G. Schmitt, "Multiscaling and joint multiscaling description of the atmospheric wind speed and the aggregate power output from a wind farm," Nonlinear Processes Geophys. 21(2), 379-392 (2014).

${ }^{7}$ N. Ali, A. S. Aseyev, and R. B. Cal, "Structure functions, scaling exponents and intermittency in the wake of a wind turbine array," J. Renewable Sustainable Energy 8(1), 013304 (2016).

${ }^{8}$ H. Kadum, S. Rockel, M. Hölling, J. Peinke, and R. B. Cal, "Wind turbine wake intermittency dependence on turbulence intensity and pitch motion," J. Renewable Sustainable Energy 11(5), 053302 (2019).

${ }^{9}$ S. Boersma, B. M. Doekemeijer, P. M. O. Gebraad, P. A. Fleming, J. Annoni, A. K. Scholbrock, J. A. Frederik, and J.-W. van Wingerden, "A tutorial on control-oriented modeling and control of wind farms," in 2017 American Control Conference (ACC) (IEEE, 2017), pp. 1-18.

${ }^{10}$ S. L. Brunton, B. W. Brunton, J. L. Proctor, E. Kaiser, and J. N. Kutz, "Chaos as an intermittently forced linear system," Nat. Commun. 8(1), 19 (2017).

${ }^{11}$ I. Mezić, "Spectral properties of dynamical systems, model reduction and decompositions," Nonlinear Dyn. 41(1-3), 309-325 (2005).

${ }^{12}$ D. Giannakis and A. J. Majda, "Nonlinear Laplacian spectral analysis for time series with intermittency and low-frequency variability," Proc. Nat. Acad. Sci. 109(7), 2222-2227 (2012).

${ }^{13} \mathrm{D}$. Giannakis, "Data-driven spectral decomposition and forecasting of ergodic dynamical systems," Appl. Comput. Harmonic Anal. 47, 338 (2019).

${ }^{14} \mathrm{H}$. Arbabi and I. Mezic, "Ergodic theory, dynamic mode decomposition, and computation of spectral properties of the Koopman operator," SIAM J. Appl, Dyn. Syst. 16(4), 2096-2126 (2017)

${ }^{15} \mathrm{M}$. Korda and I. Mezić, "Linear predictors for nonlinear dynamical systems: Koopman operator meets model predictive control," Automatica 93, 149-160 (2018).

${ }^{16}$ D. Giannakis, A. Kolchinskaya, D. Krasnov, and J. Schumacher, "Koopman analysis of the long-term evolution in a turbulent convection cell," J. Fluid Mech. 847, 735-767 (2018).

${ }^{17}$ M. A. Khodkar, P. Hassanzadeh, and A. Antoulas, “A Koopman-based framework for forecasting the spatiotemporal evolution of chaotic dynamics with nonlinearities modeled as exogenous forcings," arXiv:1909.00076 (2019).
${ }^{18}$ P. M. O. Gebraad, F. W. Teeuwisse, J.-W. van Wingerden, P. A. Fleming, S. D. Ruben, J. R. Marden, and L. Y. Pao, "A data-driven model for wind plant power optimization by yaw control," in 2014 American Control Conference (IEEE, 2014), pp. 3128-3134.

${ }^{19}$ G. V. Iungo, F. Viola, U. Ciri, M. A. Rotea, and S. Leonardi, "Data-driven RANS for simulations of large wind farms," J. Phys. 625, 012025 (2015).

${ }^{20} \mathrm{G}$. V. Iungo, C. Santoni-Ortiz, M. Abkar, F. Porté-Agel, M. A. Rotea, and S. Leonardi, "Data-driven reduced order model for prediction of wind turbine wakes," J. Phys. 625, 012009 (2015).

${ }^{21}$ J. Park, S.-D. Kwon, and K. Law, "A data-driven, cooperative approach for wind farm control: A wind tunnel experimentation," Energies 10(7), 852 (2017).

${ }^{22} \mathrm{~T}$. Göçmen and G. Giebel, "Data-driven wake modelling for reduced uncertainties in short-term possible power estimation,” J. Phys. 1037, 072002 (2018).

${ }^{23} \mathrm{X}$. W. Ti, Z. Deng, and H. Yang, "Wake modeling of wind turbines using machine learning," Appl. Energy 257, 114025 (2020).

${ }^{24}$ J. L. Proctor, S. L. Brunton, and J. N. Kutz, "Dynamic mode decomposition with control,” SIAM J. Appl. Dyn. Syst. 15(1), 142-161 (2016).

${ }^{25}$ R. B. Cal, J. Lebrón, L. Castillo, H. S. Kang, and C. Meneveau, "Experimental study of the horizontally averaged flow structure in a model wind-turbine array boundary layer," J. Renewable Sustainable Energy 2(1), 013106 (2010).

${ }^{26} \mathrm{~J} .-\mathrm{N}$. Juang and R. S. Pappa, "An eigensystem realization algorithm for modal parameter identification and model reduction,” J. Guid., Control, Dyn. 8(5), 620-627 (1985).

${ }^{27}$ D. S. Broomhead, R. Jones, M. Fleischmann, D. J. Tildesley, and R. C. Ball, “Time-series analysis," Proc. R. Soc. London, Ser. A 423(1864), 103-121 (1989).

${ }^{28}$ N. Platt, E. Spiegel, and C. Tresser, “On-off intermittency: A mechanism for bursting,” Phys. Rev. Lett. 70(3), 279 (1993).

${ }^{29} \mathrm{~N}$. Ali and R. B. Cal, "Scale evolution, intermittency and fluctuation relations in the near-wake of a wind turbine array," Chaos, Solitons Fractals 119, 215-229 (2019).

${ }^{30}$ N. Ali, A. S. Aseyev, M. S. Melius, M. Tutkun, and R. B. Cal, "Evaluation of higher order moments and isotropy in the wake of a wind turbine array," Whither Turbulence and Big Data in the 21st Century? (Springer, 2017), pp. 273-292.

${ }^{31}$ N. Ali, N. Hamilton, G. Cortina, M. Calaf, and R. B. Cal, "Anisotropy stress invariants of thermally stratified wind turbine array boundary layers using large eddy simulations," J. Renewable Sustainable Energy 10(1), 013301 (2018).

${ }^{32}$ N. Ali, H. F. Kadum, and R. B. Cal, "Focused-based multifractal analysis of the wake in a wind turbine array utilizing proper orthogonal decomposition," J. Renewable Sustainable Energy 8(6), 063306 (2016).

${ }^{33}$ S. Rockel, J. Peinke, M. Hölling, and R. B. Cal, "Wake to wake interaction of floating wind turbine models in free pitch motion: An eddy viscosity and mixing length approach," Renewable Energy 85, 666-676 (2016).

${ }^{34}$ S. Rockel, E. Camp, J. Schmidt, J. Peinke, R. B. Cal, and M. Hölling, "Experimental study on influence of pitch motion on the wake of a floating wind turbine model," Energies 7(4), 1954-1985 (2014).

${ }^{35}$ S. Rockel, J. Peinke, M. Hölling, and R. B. Cal, "Dynamic wake development of a floating wind turbine in free pitch motion subjected to turbulent inflow generated with an active grid," Renewable Energy 112, 1-16 (2017). 